\title{
Schildmann, Ulrike
}

\section{Von der (reflexiven) Koedukation zur (reflexiven) Inklusion. Ein Hürdenlauf der besonderen Art}

Budde, Jürgen [Hrsg.]; Offen, Susanne [Hrsg.]; Tervooren, Anja [Hrsg.]: Das Geschlecht der Inklusion. Opladen ; Berlin ; Toronto : Verlag Barbara Budrich 2016, S. 75-95. - (Jahrbuch Frauen- und Geschlechterforschung in der Erziehungswissenschaft; 12)

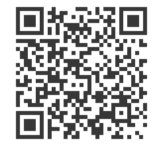

Quellenangabe/ Reference:

Schildmann, Ulrike: Von der (reflexiven) Koedukation zur (reflexiven) Inklusion. Ein Hürdenlauf der besonderen Art - In: Budde, Jürgen [Hrsg.]; Offen, Susanne [Hrsg.]; Tervooren, Anja [Hrsg.]: Das Geschlecht der Inklusion. Opladen ; Berlin ; Toronto : Verlag Barbara Budrich 2016, S. 75-95 - URN: urn:nbn:de:0111-pedocs-157537 - DOI: 10.25656/01:15753

https://nbn-resolving.org/urn:nbn:de:0111-pedocs-157537

https://doi.org/10.25656/01:15753

in Kooperation mit / in cooperation with:

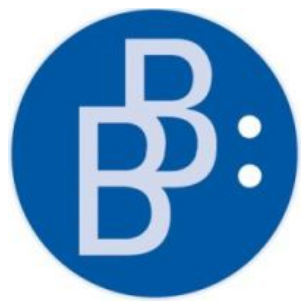

https://www.budrich.de

\section{Nutzungsbedingungen}

Gewährt wird ein nicht exklusives, nicht übertragbares, persönliches und beschränktes Recht auf Nutzung dieses Dokuments. Dieses Dokument ist ausschließlich für den persönlichen, nicht-kommerziellen Gebrauch bestimmt. Die Nutzung stellt keine Übertragung des Eigentumsrechts an diesem Dokument dar und gilt vorbehaltlich der folgenden Einschränkungen: Auf sämtlichen Kopien dieses Dokuments müssen alle Urheberrechtshinweise und sonstigen Hinweise auf gesetzlichen Schutz beibehalten werden. Sie dürfen dieses Dokument nicht in irgendeiner Weise abändern, noch dürfen Sie dieses Dokument für öffentliche oder kommerzielle Zwecke vervielfältigen, öffentlich ausstellen, aufführen, vertreiben oder anderweitig nutzen.

Mit der Verwendung dieses Dokuments erkennen Sie die Nutzungsbedingungen an.

\section{Terms of use}

We grant a non-exclusive, non-transferable, individual and limited right to using this document.

This document is solely intended for your personal, non-commercial use. Use of this document does not include any transfer of property rights and it is conditional to the following limitations: All of the copies of this documents must retain all copyright information and other information regarding legal protection. You are not allowed to alter this document in any way, to copy it for public or commercial purposes, to exhibit the document in public, to perform, distribute or otherwise use the document in public.

By using this particular document, you accept the above-stated conditions of use.

\section{Kontakt / Contact:}

\section{peDOCS}

DIPF | Leibniz-Institut für Bildungsforschung und Bildungsinformation Informationszentrum (IZ) Bildung

E-Mail: pedocs@dipf.de

Internet: www.pedocs.de

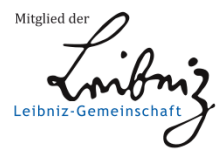


Das Geschlecht der Inklusion 
Jahrbuch

Frauen- und Geschlechterforschung

in der Erziehungswissenschaft

Redaktion

Jürgen Budde

Vera Moser

Barbara Rendtorff

Christine Thon

Katharina Walgenbach

Beirat

Birgit Althans

Sabine Andresen

Eva Breitenbach

Rita Casale

Bettina Dausien

Isabell Diehm

Hannelore Faulstich-Wieland

Edgar Forster

Edith Glaser

Carola Iller

Marita Kampshoff

Margret Kraul

Andrea Liesner

Susanne Maurer

Astrid Messerschmidt

Inga Pinhard

Annedore Prengel

Folge 12/2016 
Jürgen Budde

Susanne Offen

Anja Tervooren (Hrsg.)

\section{Das Geschlecht der Inklusion}

Verlag Barbara Budrich

Opladen • Berlin • Toronto 2016 
Bibliografische Information der Deutschen Nationalbibliothek

Die Deutsche Nationalbibliothek verzeichnet diese Publikation in der Deutschen

Nationalbibliografie; detaillierte bibliografische Daten sind im Internet über

http://dnb.d-nb.de abrufbar.

Gedruckt auf säurefreiem und alterungsbeständigem Papier.

Alle Rechte vorbehalten.

C 2016 Verlag Barbara Budrich, Opladen, Berlin \& Toronto

www.budrich-verlag.de

ISBN (Paperback) 978-3-8474-0794-2

eISBN (eBook) 978-3-8474-0922-9

Das Werk einschließlich aller seiner Teile ist urheberrechtlich geschützt. Jede Verwertung außerhalb der engen Grenzen des Urheberrechtsgesetzes ist ohne Zustimmung des Verlages unzulässig und strafbar. Das gilt insbesondere für Vervielfältigungen, Übersetzungen, Mikroverfilmungen und die Einspeicherung und Verarbeitung in elektronischen Systemen.

Typographisches Lektorat: Ulrike Weingärtner, Gründau

Umschlaggestaltung: disegno visuelle kommunikation, Wuppertal - www.disenjo.de Druck: paper \& tinta, Warschau

Printed in Europe 


\section{Inhaltsverzeichnis}

Jürgen Budde, Susanne Offen und Anja Tervooren

Das Geschlecht der Inklusion - eine Einleitung........................... 7

\section{Teil I: Essays}

Carla Di Georgio

Mothers' and Fathers' disparate experiences in Francophone Schools: The connection between language, social capital and power in accessing 'inclusive' spaces for their children .......... 15

David Mitchell, Sharon Snyder und Linda Ware

Curricular Cripistemologies: The Crip/Queer Art of Failure ... 37

Bettina Kleiner, Torben Rieckmann und André Zimpel

Diskurstheoretische Perspektiven auf Behinderung, Geschlecht und Sexualität als mögliche Grundlage der Debatte über Inklusion. Ein Versuch ............................................................. 55

Ulrike Schildmann

Von der (reflexiven) Koedukation zur (reflexiven) Inklusion ein Hürdenlauf der besonderen Art.......................................... 75 


\section{Teil II: Thementeil}

Jürgen Budde und Nina Blasse

Vergeschlechtlichungen von Care im inklusiven Unterricht.... 99

Heike Raab

Re/Visionen - Inklusion, Behinderung und Geschlecht

Mechthild Bereswill und Johanna Zühlke

„Faktor Frau kommt meilenweit danach“. Eine qualitative

Exploration zum Verhältnis von Geschlecht und Behinderung

\section{Teil III: Offener Teil}

Margarete Menz und Christine Thon

Familie und Beruf - oder? Hegemoniale Diskurse,

(un)zureichende Alternativen und die Suche nach dem ,guten

Leben"

Autor*innen 


\title{
Von der (reflexiven) Koedukation zur (reflexiven) Inklusion ${ }^{1}$ - ein Hürdenlauf der besonderen Art
}

\author{
Ulrike Schildmann
}

\section{Ausgangspunkt I: Reflexive Koedukation}

Seit Beginn der 1990er Jahre wird in Deutschland der erziehungswissenschaftliche Diskurs über die gemeinsame Erziehung und Bildung von Jungen und Mädchen unter dem Stichwort der „reflexiven Koedukation“ (FaulstichWieland 1994) geführt. Bis dahin hatte er bereits eine fast 100jährige Geschichte durchlaufen (vgl. Kreienbaum 2004; Popp 2011; Schildmann 2011): Begonnen hatte die Auseinandersetzung um Koedukation Anfang des 20. Jahrhunderts im Zuge des Kampfes um die höhere Mädchenbildung. Er war über Jahrzehnte hinweg zunächst fast nur von engagierten Frauen geführt worden. Nach dem Zweiten Weltkrieg erlebte die Koedukation unterschiedliche Entwicklungen in Ost und West. In der Deutschen Demokratischen Republik (DDR) galt die gemeinsame Erziehung (auch die höhere Bildung) der Jungen und Mädchen - im ideologischen und institutionellen Rahmen des sozialistischen Bildungswesens - von Anfang an als Selbstverständlichkeit. In der (alten) Bundesrepublik Deutschland (BRD) wurde sie dagegen erst im Zuge der ersten großen Strukturreform des Bildungswesens - 1965 eingeführt: Mit ihr sollten alle Hindernisse gegen die formale Gleichstellung von Jungen und Mädchen in der Bildung beseitigt werden. Sie war in der Entstehungsphase der 1960er Jahre vor allem bildungspolitisch motiviert und entging deshalb zunächst einer intensiveren (auch kritischen) erziehungswissenschaftlichen Reflexion. Eine solche setzte erst mit Entstehen feministischer Lehrerinnengruppen und der sozial- bzw. erziehungswissenschaftlichen Frauenforschung um 1980 ein und ist seitdem Bestandteil der feministischen Schul- und Bildungsforschung in allen Bereichen und auf allen Stufen des Bildungswesens. „Reflexive Koedukation“ umschrieb Hannelore FaulstichWieland im ,Jahrbuch für Pädagogik 1994“ wie folgt:

1 Da der vorliegende Beitrag für ein Jahrbuch Frauen- und Geschlechterforschung in der Erziehungswissenschaft konzipiert wurde, beziehen sich seine Überlegungen und Ausführungen zum Thema Inklusion weitestgehend auf die Inklusive Pädagogik und nicht, wie dies auch möglich wäre, auf allgemeine gesellschaftliche Prozesse von Inklusion und Exklusion. 
„Vordringlich sind ... Maßnahmen zur Sensibilisierung von Lehrerinnen und Lehrern. Die Reflexion darüber, welche Vorstellungen von 'Weiblichkeit' und 'Männlichkeit' bei einem selbst vorhanden sind, wie man selbst die Geschlechterverhältnisse sieht, erlaubt zunächst einmal, überhaupt wahrzunehmen, ob und wie sich Benachteiligungen für Mädchen, aber auch für Jungen herstellen. Solche Reflexionen zu ermöglichen und darauf aufbauende Verhaltensänderungen zu unterstützen, halte ich für ein zentrales strategisches Moment in das die Trennungsfrage (im Sinne von Monoedukation - U.Sch.) nur eingebettet sein, das aber nicht auf ihr aufbauen kann“ (Faulstich-Wieland 1994: 338).

Knapp 20 Jahre später schlägt die Begründerin der „,reflexiven Koedukation“ selbst - hier gemeinsam mit Marianne Horstkemper - im Rahmen einer kritischen Durchsicht von „Forschungen zur reflexiven Koedukation, also zum Versuch, koedukative wie monoedukative Kontexte auf die zugrunde liegenden Geschlechterverhältnisse rückzubeziehen“" (Faulstich-Wieland \& Horstkemper 2012: 34), folgende Differenzierung vor:

„Alle genannten Forschungen verdeutlichen, dass Dramatisierungen der Differenzen durch die klare Unterscheidung der Geschlechter zum einen die Wahrnehmung der Differenzierungen innerhalb der Gendergruppen erschweren, zum anderen befördern sie mindestens teilweise ein stereotypes doing gender durch die Schülerinnen und Schüler.“

Die Forderung nach Entdramatisierungen von Geschlecht kann allerdings nicht bedeuten, zur vermeintlichen Geschlechtsneutralität zurück zu gehen. Jene Lehrkräfte, die glauben, keine Geschlechterunterschiede zu machen, sondern alle gleich zu behandeln, sind sehr wohl in die alltäglichen doing gender Prozesse involviert... Sie reproduzieren auf diese Weise durchaus die 'normalen' Geschlechterbilder. Pädagogisches Handeln wäre deshalb rückzubinden an Selbstreflexionen des eigenen ,doing gender“ (ebd.; vgl. auch Schildmann 2012; zu den 'normalen' Geschlechterbildern vgl. Kapitel 3 dieses Beitrags).

Der Begriff der „reflexiven Koedukation“ einschließlich der Schrittfolge von Dramatisierung, Reflexion und Entdramatisierung hat sich, trotz der genannten Differenzierungsnotwendigkeit, zur Bezeichnung erziehungswissenschaftlicher Bemühungen um eine geschlechtergerechte Pädagogik inzwischen weitgehend etabliert (vgl. Kampshoff \& Wiepcke 2012: $1 \mathrm{f}^{2}$ ). Mehr noch: Er dient sogar als Ausgangspunkt für weitere, ähnliche Begriffskombinationen, so vor allem ,,reflexive Interkulturalität“ und schließlich auch ,reflexive Inklusion“, wie bei Jürgen Budde und Merle Hummrich (2013) zusammenfassend zu lesen ist:

2 In ihrer Einführung in das 2012 erschienene „Handbuch Geschlechterforschung und Fachdidaktik“ schreiben die Herausgeberinnen, Marita Kampshoff und Claudia Wiepcke: „Für eine Umsetzung von Geschlechtergerechtigkeit schlagen Faulstich-Wieland u.a. (2008: 11ff.) einen Dreischritt vor: Dramatisierung - Reflektion (sic!) - Entdramatisierung“ (Kampshoff \& Wiepcke 2012: 1. Ausführungen zu den drei Analyseschritten folgen in direktem Anschluss (1f.)). 
„In Bezug auf die sozialen Kategorien Gender und Ethnizität haben in den letzten Jahrzehnten als zugehörige pädagogische Konzepte die reflexive Koedukation bzw. reflexive Interkulturalität an Bedeutung gewonnen (vgl. Faulstich-WielandHorstkemper 1995; Hamburger 2000). In beiden Konzepten ist ein Spannungsfeld aus Dramatisierung und Entdramatisierung sozialer Differenzkategorien angelegt... Angelehnt an diese Konzepte schlagen wir für das hier diskutierte Thema vor, die Perspektive hin zu einer reflexiven Inklusion zu wechseln“ (Budde \& Hummrich 2012: 4).

Damit wäre Ausgangspunkt I des vorliegenden Beitrages umrissen: Der Begriff der reflexiven Inklusion wurde - von Budde und Hummrich 2013 - auf der Basis der reflexiven Koedukation, und nachfolgend der reflexiven Interkulturalität, in den erziehungswissenschaftlichen Diskurs eingeführt.

\section{Ausgangspunkt II: Integrationspädagogik und ihre Geschlechterdimension (Koedukation in der Integrationspädagogik)}

Ausgangspunkt dieses Kapitels ist der weit verbreitete Eindruck, Bildungspolitik und -praxis konzentrierten sich beim Thema Inklusion häufig nur auf die Kategorie Behinderung, statt die unterschiedlichen sozialen Ungleichheitslagen und deren Verbindungen miteinander zu reflektieren. So beklagen Jürgen Budde und Merle Hummrich:

„Inklusion scheint in der gegenwärtigen bildungspolitischen und -praktischen Diskussion allgegenwärtig. Im Kern wird dabei bislang zumeist aus einer sonder- und rehabilitationspädagogischen Perspektive die Anerkennung bzw. die Teilhabe von Menschen mit Behinderungen gefordert $[\ldots]$ andere Ungleichheitsdimensionen scheinen demgegenüber in den Hintergrund zu geraten" (Budde \& Hummrich 2013: 1).

Warum dieser Eindruck Platz gegriffen hat, obwohl die inklusive Pädagogik weit über die Belange behinderter Menschen hinausgeht und stattdessen auf die gemeinsame Erziehung und Bildung aller Kinder und Jugendlichen gerichtet ist, soll in einem, dem eigentlichen Inhalt dieses Kapitels vorangestellten Exkurs erläutert werden:

\section{Exkurs:}

Den Anstoß für die öffentlichkeitswirksame Inklusionsdebatte der letzten Jahre, insbesondere für die Einbeziehung behinderter Kinder und Jugendli- 
cher in allgemeine Schulen, gab die UN-Konvention über die Rechte von Menschen mit Behinderungen (UN-BRK). Mit dieser Konvention wurde im Gesamtrahmen der UN-Menschenrechte eine Lücke geschlossen: Wurde eine Konvention zur Beseitigung rassistischer Diskriminierung 1965 verabschiedet, die Frauenrechtskonvention 1981, die Kinderrechtskonvention 1989, und außerdem 1989 eine allgemeine Formulierung von Diskriminierung (in der Behinderung nicht gesondert ausgewiesen, sondern unter „,sonstiger Status“ subsummiert wurde; vgl. Prasad 2015: 130f.), so erfolgte die Verabschiedung der Behindertenrechtskonvention erst 2006 (Ratifizierung durch Deutschland 2009).

Dieser Konvention waren seit den 1990er Jahren verschiedene Debatten einzelner UN-Organisationen vorausgegangen, u.a. solche der UNESCO, die sich mit den internationalen Perspektiven der Special Needs Education auseinandersetzten und deshalb die Kategorie Behinderung fokussierten. Florian Kiuppis kommt in seiner Analyse von UNESCO-Schlüsseltexten - hier der Salamanca-Erklärung von 1994 - zu folgender Aussage:

„Special Needs Education, so wird in diesem Dokument ausgeführt, könne nicht in Isolation fortgeführt werden und müsse folglich unter dem Vorzeichen des neuen Leitprinzips der Inklusion in Richtung der im Jahr 1990 ins Leben gerufenen globalen bildungspolitischen Education for All-Agenda umorientiert werden, 'to ensure that Education for All means FOR ALL, particular those who are most vulnarable and most in need (UNESCO 1994: S. iv_Hervorhebung im Original) '“ (Kiuppis 2014: 131).

In ihren „Leitlinien für die Bildungspolitik“ verweist die Deutsche UNESCO-Kommission ,,auf die Möglichkeit, in inklusiven Einrichtungen beim Spielen und Lernen auf individuelle Unterschiede einzugehen - zum Vorteil aller Kinder" (Platte 2015: 131 in Anlehnung an die Deutsche UNESCOKommission 2010), nicht nur der behinderten, denn die Einstellung zu menschlicher Vielfalt sei eine Sache aller und könne „nur im gemeinsamen Spielen und Lernen wachsen“"(ebd.).

Wenn sich dennoch in der deutschen Bildungspolitik der Eindruck aufdrängt, Inklusion meine vor allem die behinderten Kinder und Jugendlichen, dann ist dies nicht verwunderlich, geht es doch a) auf der institutionellen Ebene u.a. auch um die Zukunft (Erhalt oder Auflösung) eines höchst differenzierten Sonder-/Förderschulsystems im Rahmen des hierarchischen deutschen Schulsystems und b) auf der professionellen Ebene um unterschiedliche Positionen der beteiligten Pädagog*innen, unter denen sich - auf beiden Seiten des Spielfeldes - die wenigsten für die neuen Herausforderungen gut vorbereitet fühlen. Und wenn, wie Budde und Hummrich beobachten, „bislang zumeist aus einer sonder- und rehabilitationspädagogischen Perspekti-

3 „Die UNESCO-Kommission nennt als Begründung für eine Schule für alle schließlich die geringeren Kosten 'im Vergleich zu einem komplexen System unterschiedlicher Schultypen'“(Platte 2015: 131). 
ve" (s.o.) verhandelt wird, dann geschieht dies m.E. vor allem deshalb, weil der bildungspolitische Druck derzeit auf einer neuen institutionellen Positionierung der Schüler*innen mit sonderpädagogischem Förderbedarf im Schulwesen liegt, für die sich vor allem Sonder- und Rehabilitationspädagog*innen zuständig erklären, während sich die Pädagog*innen der allgemeinen Schulen vergleichsweise abwartend verhalten und sich für die neuen Aufgaben tendenziell unvorbereitet fühlen.

Auffällig und gewissermaßen verwunderlich ist im Zusammenhang mit der Einführung der inklusiven Pädagogik, dass in den derzeitigen bildungspolitischen Kontroversen die früheren Praxismodelle und theoretischen Ansätze der Integrationspädagogik, d.h. die gemeinsame Erziehung und Bildung behinderter und nicht behinderter Kinder und Jugendlicher, von der breiten (Fach-)Öffentlichkeit kaum zu Rate gezogen werden, wie z.B. Andrea Platte feststellt:

„Wenig zur Kenntnis genommen werden dabei die im deutschsprachigen Raum seit den 1970er Jahren bestehenden pädagogischen Bestrebungen in Richtung des gemeinsamen Spielens und Lernens von behinderten und nicht behinderten Kindern, des gemeinsamen Unterrichts, der Bildung ohne Aussonderung mit wissenschaftlichen Begleitungen, vor allem im Elementar- und Primarbereich“ (Platte 2015: 131).

Diese Einschätzung wird z.B. von Ulf Preuss-Lausitz (2011) in einem Überblicksartikel über „Integration und Inklusion von Kindern mit Behinderungen - Ein Weg zur produktiven Vielfalt in einer gerechten Schule“ untermauert:

„Wurde seit den 1970er Jahren im deutschen Sprachgebrauch von 'Integration` gesprochen und war damit die gemeinsame Erziehung in der Klasse (und nicht etwa in einer Sonderklasse der Regelschule) gemeint, beginnt Mitte der 2000er Jahre in der Fachdiskussion die Adaption des Begriffs 'Inklusion' aus dem englischen Sprachgebrauch (vgl. Schnell \& Sander 2004). [...] Falsch wäre es, die über dreißigjährige integrationspädagogische Praxis dadurch abzuwerten, dass 'Integration` als additive Praxis (etwa in separierten Räumen), 'Inklusion' jedoch als wahre gemeinsame Erziehung angesehen würde" (Preuss-Lausitz 2011: 169f.).

In der Theorieentwicklung waren sich die an der Integrationspädagogik Beteiligten - trotz unterschiedlicher Theorieansätze - seit den 1980er Jahren darin einig, dass es um eine neue Pädagogik für alle Kinder, nicht nur für behinderte, gehen müsse. So formulierte etwa Georg Feuser:

„Integrationspädagogik bedarf einer Pädagogik, in der

- alle Kinder

- $\quad$ in Kooperation miteinander

- auf ihrem jeweiligen Entwicklungsniveau

- $\quad$ an und mit einem gemeinsamen Gegenstand spielen und lernen“ (Feuser 1986: 129).

In diesem Sinne entwarf Georg Feuser im Laufe der 1980er Jahre eine „Allgemeine integrative Pädagogik und entwicklungslogische Didaktik“ (Feuser 
1989) mit einer umfassenden, weit über behinderungsspezifische Anliegen hinausgehenden Theoriekonzeption.

Für eine zu entwickelnde reflexive Inklusionspädagogik, die auf die unterschiedlichen sozialen Ungleichheitslagen - und hier vor allem auf die Kategorie Geschlecht - eingeht, wurden entsprechende Differenzierungen im integrationspädagogischen Diskurs spätestens seit Beginn der 1990er Jahre sichtbar:

- Intensiv erarbeitet wurde die Verbindung zwischen den (Reform)Strömungen der feministischen, interkulturellen und integrativen Pädagogik (vgl. Prengel 1993a; aber auch Hinz 1993; Wenning 1999), wobei vor allem die Entwicklung einer „Pädagogik der Vielfalt“ durch Annedore Prengel bis heute nachhaltige Wirkung zeigt (vgl. zuletzt ein 2015 publiziertes Schwerpunktheft der Zeitschrift „Erwägen Wissen Ethik“ mit Hauptartikel von Prengel und 45 daran orientierten wissenschaftlichen Stellungnahmen).

- Gezielt erarbeitet wurden darüber hinaus (auf der Basis von Annedore Prengels Vergleich der o.g. drei pädagogischen Reformbewegungen) zentrale Zusammenhänge zwischen „Integrationspädagogik und $\mathrm{Ge}$ schlecht" (vgl. Schildmann 1996). Auf vier analytischen Ebenen (der individuellen, interaktiven, institutionellen und gesellschaftlichen Ebene) wurden die Positionen der behinderten und nicht behinderten Mädchen und Jungen in der integrativen Pädagogik, ihrer Mütter und Väter sowie der an der Integrationspädagogik beteiligten Berufsgruppen (Erzieher*innen und Lehrer*innen) auf der Basis vorliegender Forschungsergebnisse analysiert, um so die „Geschlechterdimension in der Integrationspädagogik" theoretisch zu erfassen und für eine integrationsbezogene Didaktik nutzbar zu machen. ${ }^{4}$

- Nicht zuletzt wurden Fragen der Koedukation in der Integrationspädagogik erörtert. Verwiesen sei an dieser Stelle vor allem auf den Fachdiskurs zwischen Ulf Preuss-Lausitz und Annedore Prengel, basierend auf deren Artikel mit dem provokativen Titel: „Sind Mädchen die Integrationshelferinnen par excellence?“ (1993b), gegen den Ulf Preuss-Lausitz (1998) konterte mit „Auch Behinderte sind Jungen und Mädchen“. Die Auseinandersetzung basierte auf empirischen Daten unterschiedlicher Art (Auswertung von Lehrer*innen-Beobachtungen im Unterricht versus Schüler*innen-Befragungen) sowie auf divergierenden theoretischen Ansätzen der Sozialisation der Geschlechter (feministische Theorie mit Schwerpunktsetzung auf Unterschieden zwischen Mädchen und Jungen versus soziologische Modernisierungstheorie mit Schwerpunktsetzung auf Unterschieden innerhalb jedes einzelnen Geschlechts).

4 Für Zusammenhänge zwischen Integrationspädagogik und interkultureller Pädagogik (vgl. Merz-Atalik 2001). 
Die Beispiele zeigen, dass Koedukation nicht nur in der allgemeinen Pädagogik (wie auch in der Sonderpädagogik: vgl. dazu exemplarisch Rohr 1980; Moser et al. 2006) Thema war und ist, sondern auch in der Integrationspädagogik, weshalb auch deren Ansätze in die Überlegungen zu einer ,,reflexiven Inklusion" einbezogen werden sollten. Grundsätzlich bedacht werden sollte an dieser Stelle, dass die inklusive Pädagogik über eine Vorläuferin verfügt, an deren wissenschaftliche Theoriearbeit sowie Projekterfahrungen angeknüpft werden kann.

\section{Theoretischer Hintergrund I: Normalismus in der Bildung}

Im pädagogischen Diskurs der letzten Jahre ist immer häufiger die Rede von einem reflexiven Umgang mit Heterogenität. Für die einen resultiert diese Orientierung aus den formulierten Zielsetzungen der integrativen/inklusiven Pädagogik, die nur erreicht werden könnten, wenn die Verschiedenheit der zu erziehenden Kinder und Jugendlichen wertschätzend akzeptiert und zum Ausgangspunkt aller pädagogischen Handlungen genommen werde, für die anderen resultiert sie (schlicht) aus der Erkenntnis, dass die nachwachsenden Generationen mehr durch individuelle Verschiedenheit (Heterogenität) als durch Gleichheit (Homogenität) gekennzeichnet seien, und sich die Pädagogik deshalb mehr auf die Heterogenität der Schülerschaft einstellen müsse. Schwierig scheint diese Umorientierung vor allem dadurch zu sein, dass das deutsche Bildungssystem als solches ,strukturell auf Homogenisierung der Schülerschaft ausgerichtet" ist (Faulstich-Wieland 2011: 9; vgl. dazu grundlegend auch Wenning 1999). Das belegt die Leistungsdifferenzierung, die vor allem - im Anschluss an die gemeinsame Grundschulzeit für (fast) alle Kin$\operatorname{der}^{5}$ - das Sekundarschulwesen kennzeichnet: Hauptschule, Realschule, Gesamtschule, Gymnasium und nicht zu vergessen ein differenziertes Sonderschulsystem stellen die traditionelle Struktur des Schulwesens dar, die erst langsam aufgebrochen wird. Die strukturelle Leistungsorientierung - gepaart mit dem Jahrgangsprinzip - wird zunehmend, u.a. durch die internationalen Vergleichsstudien (PISA u.Ä.), in Frage gestellt; denn diese haben gezeigt, „dass es ein breites Überlappungsfeld in den Leistungen zwischen diesen

5 Ein kleiner Teil der Schulanfänger*innen wird jedoch von Anfang an in Förderschulen geschickt. Im Jahr 2010 wurden 3,4 \% aller Kinder direkt in eine Förderschule eingeschult, also ohne jeden schulischen Integrationsversuch, und zwar 4,5\% der Jungen und 2,3\% der Mädchen (vgl. Autorengruppe Bildungsberichterstattung 2012: 63). Diese Schieflage deutet sich schon unter den Empfängern von Eingliederungshilfe im vorschulischen Alter an (vgl. Marks 2011; Schildmann 2011a). 
Schularten gibt" (Faulstich-Wieland 2011: 9). Die strukturellen Bedingungen würden, so die Autorin, „keineswegs eine Homogenität der Schülerschaft bewirken“ (ebd.), vielmehr handele es sich in allen Schularten um heterogene Zusammensetzungen. In kritischer Absicht fährt sie fort:

„Die pädagogische Maxime, sich dennoch an den 'Mittelköpfen` (Sandfuchs 2004: 490 ${ }^{6}$ ) zu orientieren, führt zu Friktionen und zu bildungspolitisch nicht vertretbaren Ungleichheiten. Hinzu kommt, dass die Frage, was denn die 'Mittelköpfe' charakterisiere, nicht nur leistungsmäßig kaum verlässlich zu klären ist, sondern in Bezug auf weitere Merkmale der Schülerinnen und Schüler (genannt werden kulturelle und soziale Herkunft sowie Geschlecht - U.Sch.) kein lineares Maß ergibt" (Faulstich-Wieland 2011: 10).

Die Notwendigkeit zur kritischen Auseinandersetzung mit der Orientierung an der statistischen Normalverteilung und mit Leistung als zentralem Basisnormalfeld und Ordnungskriterium der schulischen Organisation wird hier augenfällig. Mehr noch: Deutlich wird die Notwendigkeit, einen kritischen Diskurs nicht nur darüber zu führen, welche Abweichungsformen von der Normalität existieren und wie diese definiert werden, sondern darüber, was im deutschen Bildungswesen unter Normalität selbst verstanden wird, wie Normalität hergestellt wird (vgl. den Titel des Hauptwerkes von Jürgen Link 1997) und schließlich, welche Formen der Flexibilisierung von Normalität bzw. Überwindung normalistischer Strukturen und Strategien im Bildungswesen für denkbar und durchsetzbar gehalten werden.

Für die Integrationspädagogik liegen dazu systematische normalismustheoretrische Inhaltsanalysen vor (vgl. Lingenauber 2003; Schildmann 2004) ${ }^{7}$. Sie konzentrieren sich exemplarisch auf die Publikationen der Integrationspädagogen Hans Eberwein, Georg Feuser und Annedore Prengel zwischen 1970 und 2000. Gerade an dem Werk von Annedore Prengel konnte die zentrale Bedeutung des Basisnormalfeldes Leistung im Zusammenhang mit Behinderung und Geschlecht herausgearbeitet werden. An den Schriften aller drei Autoren konnte darüber hinaus gezeigt werden, dass auf dem Feld der Integrationspädagogik eine Diskursstrategie verfolgt wurde, die mit dem Begriff Transnormalismus zu bezeichnen ist und sich damit diskursiv - wie auch die Konzeption der inklusiven Pädagogik - jenseits des Normalismus ansiedelt. Als Transnormalismus bezeichnet Jürgen Link ein nicht auf statistischen Durchschnitten fußendes Normalitätskonzept:

„Die Zonen möglicher transnormalistischer Exploration lagen und liegen stets uneindeutig und unentschieden in einer Art Schwebezustand an der Grenze des flexiblen Normalismus, in den zurück sie jederzeit ,gefloatet‘ werden konnten und können“ (Link 1997: 33).

6 „Sandfuchs schreibt diesen Vorschlag Ernst Christian Trapp (1745-1818) zu [...]“ (Faulstich-Wieland 2011: 10, Fußnote 3).

7 Vgl. DFG-Forschergruppe „Normalismus“ der TU Dortmund 1998-2001/2004, Sprecher: Prof. Dr. Jürgen Link; Teilprojekt „Leben an der Normalitätsgrenze“ - Leitung Prof. Dr. Ulrike Schildmann. 
Während sich der Normalismusforscher Link selbst auf die Strukturen und Zusammenhänge von Protonormalismus und flexiblem Normalismus konzentriert und den Transnormalismus nur am Rande berührt, sind die genannten drei Integrationspädagogen - diskursiv - geradezu auf transnormalistische Strukturen fixiert, d.h. ihnen ist daran gelegen, nicht nur den Protonormalismus, sondern auch den flexiblen Normalismus zu überwinden - und mit diesen beiden Diskursstrategien die Grundstruktur jeglicher Normalität, zumindest auf dem gesellschaftlichen Feld der Bildung (vgl. Schildmann 2009).

Auch die allgemeine Pädagogik muss sich, wenn sie einen wertschätzenden „Umgang mit Heterogenität“ im Bildungswesen verankern will, mit den Strukturen und Strategien des Normalismus auseinandersetzen. Dass sich der flexible Normalismus (mit seiner Durchlässigkeit und seinen weicheren Grenzen zwischen Normalität und Abweichung) gegenüber dem Protonormalismus (mit dessen scharfer Abgrenzung zwischen diesen) bereits im Allgemeinen durchgesetzt hat, ist keine Frage mehr - vielmehr geht es nun darum, die Flexibilitätsgrenzen näher zu analysieren, wozu es jedoch sinnvoll sein könnte, die Strukturen des Bildungswesens und seiner angrenzenden Gebiete normalismusanalytisch auszuleuchten. Dazu liegen einzelne wissenschaftliche Werke bereits vor, die mit ihren unterschiedlichen Perspektiven ein intensiver zu bearbeitendes Feld andeuten. Drei dieser Werke sollen hier vorgestellt werden:

Genannt sei an erster Stelle die Dissertation von Elisabeth von Stechow, die sich, theoretisch u.a. angelehnt an Jürgen Links Normalismusanalyse, der „Erziehung zur Normalität“ mit einer historischen Analyse „,der Ordnung und Normalisierung der Kindheit“ (2004) widmet: „Die Erziehung zur Normalität kann in modernen Gesellschaften als maßgebliches Erziehungsziel angesehen werden. Dabei sollen Kinder ein Spektrum von Verhaltensweisen ausbilden, das gemäß dem gesellschaftlichen Konsens als normal bezeichnet werden kann" (Stechow 2004: 199). Die Analyse enthält unterschiedliche Ebenen der Erziehung zur Normalität, ausgedrückt in bestimmten Strukturen von Fremdund Selbstdisziplinierung (Stechow 2004: 60 ff; 2008: 77 ff). Historisch relevant sind v.a.:

- räumliche Disziplinierung, vor allem in Haus (Familie) und Schule, verbunden mit Warngeschichten darüber, welchen Gefahren Kinder außerhalb dieser festumrissenen Räume ausgesetzt sein könnten;

- zeitliche Disziplinierung, die im Rahmen der Schule vor allem der „Herstellung einer altersgleichen Unterrichtung in leistungshomogenen Gruppen" (Stechow 2008: 81) dient, womit auch die Vorstellung von altersgemäßem Verhalten einherging;

- Disziplinierung des Körpers durch Anweisungen zur Körperhaltung, zu Mimik und Gebärden und zu angepasstem Verhalten in den vorgegebenen (Schul-)Räumen. 
Auf dieser Basis wurden Abweichungen vom kindlichen Normalverhalten definiert, die in der Pädagogik vor allem bekannt wurden unter dem Begriff der ,'Lehre von den Kinderfehlern', die Ende des 18. Jahrhunderts entstand und bis zu Beginn des 20. Jahrhunderts von Bedeutung war" (Stechow 2008: 84).

Die angerissene historische Perspektive wird durch einen von Helga Kelle und Anja Tervooren 2008 herausgegebenen Sammelband über „Ganz normale Kinder. Heterogenität und Standardisierung der Kindheit“ erweitert. Die Beiträge konzentrieren sich - historisch untermauert - auf aktuelle Diskurse zur kindlichen Entwicklung, nicht nur im schulischen Rahmen, sondern auch bereits in der vorschulischen Lebensphase. Die Herausgeberinnen weisen auf folgende Ambivalenz zwischen aktuellen Tendenzen zu gesellschaftlicher Homogenisierung und Heterogenisierung bereits in der frühkindlichen Lebensphase hin:

„Das Phänomen der Heterogenität der Bildungsvoraussetzungen der Kinder wird auf der einen Seite produktiv gewendet und in Ansätzen der Altersmischung z.B. im Rahmen der flexiblen Schuleingangsstufe sogar forciert sowie als Grundlage für die Differenzierung der Lehr- und Lernformen pädagogisch begrüßt. Auf der anderen Seite wird die Heterogenität in den expandierenden Formen der vorschulischen Beobachtung und Untersuchung der frühen kindlichen Entwicklung doch implizit auch als ein Phänomen begriffen, dem es vorzubeugen gilt. So wird wiederholt und von unterschiedlichen Professionellen [...] geprüft, ob Kinder sich 'altersgemäß' entwickeln. Kein institutioneller Bereich der Kindheit hat in der jüngsten Zeit international einen derartigen Ausbau und Institutionalisierungsschub erfahren wie die Maßnahmen zur Früherkennung und Prävention von Entwicklungsund Lernstörungen, die notwendig standardisierte Vorstellungen von altersgemäßer Entwicklung zugrunde legen (müssen)“ (Kelle \& Tervooren 2008: 7).

Während es in den Beiträgen des genannten Sammelbandes um die Konstruktionen von sogenannter normaler Kindheit geht, stehen im Zentrum einer dritten, normalismustheoretisch orientierten, Analyse die „Konstruktionen von Behinderung in den ersten Lebensjahren“" (Marks 2011). Die Autorin untersucht die Frage, auf welchem Wege bestimmte Jungen und Mädchen bereits von Geburt bzw. frühester Kindheit an als behindert identifiziert und definiert werden. Dabei wird deutlich, dass am Lebensanfang (auf Basis der standardisierten medizinischen Früherkennungsuntersuchungen) vor allem medizinische Definitionen von Normalität, Gesundheit und Krankheit ausschlaggebend für die - teils sehr vage - Identifizierung von Behinderungen sind. So wird die im ersten Lebensjahr dominierende Behinderungskategorie „übergreifend/nicht klassifizierbar“ (Marks 2011: 80) erst entlang der kindlichen Entwicklung in den folgenden Lebensjahren allmählich ausdifferenziert (nun unter Einbeziehung von Psychologie und Pädagogik) in: Lern- und geistige Behinderungen, Seh-, Sprach- und Hörbehinderungen, Körperbehinderungen, Emotionale und Soziale Störungen. In diesem Prozess findet eine 
nicht zu übersehende Dynamik der Geschlechterkonstellationen unter den betreffenden Kindern statt: „Wird am Lebensanfang bei Jungen nur eine leicht erhöhte Betroffenheit von Krankheit und gesundheitlicher Auffälligkeit festgestellt (Jungen knapp 55 Prozent, Mädchen etwa 45 Prozent), so beträgt bereits im Kindergartenalter der Jungenanteil unter den Kindern, die wegen einer Behinderung 'soziale Eingliederungshilfe' erhalten, etwa $2 / 3$ gegenüber $1 / 3$ Mädchen. Dieses quantitative Verhältnis ist auch unter den Jungen und Mädchen mit 'sonderpädagogischem Förderbedarf" im Schulalter anzutreffen" (Schildmann 2011a: 13).

Alle drei hier umrissenen Perspektiven auf die gesellschaftliche Normalität machen deutlich, wie wichtig es ist, die Argumentationsfigur des „Umgangs mit Heterogenität" in Theorie und Praxis weiterhin kritisch zu analysieren. Die Lektüre neuerer Publikationen, wie etwa des „Handbuches Geschlechterforschung und Fachdidaktik" (Kampshoff \& Wiepcke 2012) oder des in der Zeitschrift „Erwägen Wissen Ethik“ (2015) geführten FachDiskurses über die „Pädagogik der Vielfalt“ (s.o.) vermittelt den Eindruck, dass sich der Normalismus in der Bildung in den unterschiedlichen Positionen zum „Umgang mit Heterogenität“ abbildet: Die überwiegend protonormalistische Positionierung mit dem Leitziel möglichster Homogenisierung von Lerngruppen ist diskursiv noch nicht (gänzlich) überwunden. Jedoch werden flexibel normalistische Ansätze im Sinne eines pragmatischen Umgangs mit Heterogenität vermehrt vertreten und stehen inzwischen im Zentrum des erziehungswissenschaftlichen Diskurses, wogegen transnormalistische Ansätze der positiv wertschätzenden Anerkennung von Heterogenität im Sinne einer allgemeinen integrativen Pädagogik für alle Kinder (Feuser 1989 ff.), einer „Pädagogik der Vielfalt“ (Prengel 1993a: 2015) und ähnlicher (radikaler) Ansätze (wie schon zu Zeiten der Vorläuferbewegung der inklusiven Pädagogik) gegenüber den Mehrheitspositionen noch (wenn auch nun mit der UN-BRK im Rücken) erkämpft werden müssen.

\section{Theoretischer Hintergrund II: Soziale Strukturkategorien im Vergleich}

Wenn es um einen sensiblen Umgang mit Heterogenität geht, um eine (reflexive) inklusive Pädagogik zu entwickeln, dann besteht - über die kritische Auseinandersetzung mit Normalität hinaus - eine weitere Herausforderung darin, sich mit den gesellschaftlichen Strukturkategorien auseinanderzusetzen, die als relevante Indikatoren für soziale Ungleichheitslagen gelten. Dabei sind zunächst die einzelnen Kategorien in ihren jeweiligen Binnenstrukturen zu reflektieren, um aufbauend darauf mögliche Wechselwirkungen zwischen unterschiedlichen Strukturkategorien untersuchen zu können. 
„In politischen und wissenschaftlichen Diskursen erfahren einzelne soziale Strukturkategorien mehr Aufmerksamkeit als andere. In der Bildungspolitik ist auf jeden Fall eine starke Altersorientierung entlang der (statistisch) durchschnittlichen kindlichen Entwicklung (vgl. Kelle \& Tervooren 2008) zu verzeichnen. Andere Strukturkategorien - außer dem Alter finden weitgehend nur dann Berücksichtigung, wenn soziale Risiken sichtbar werden und bestimmte soziale Minderheiten-Gruppen identifiziert werden sollen. Das ist [...] bei den Kategorien Behinderung und Migrationshintergrund (Ethnizität) der Fall. Wiederum andere Kategorien, wie vor allem die Kategorien Klasse/Schicht und Geschlecht, denen sich nicht nur bestimmte, sondern alle Gesellschaftsmitglieder zuordnen lassen, bleiben hinter den hervorgehobenen Kategorien weitgehend unbeachtet, obwohl sie in entscheidendem Maße strukturierend wirken und unübersehbare Wechselwirkungen mit den herausgehobenen Kategorien eingehen“" (Schildmann 2015a: 96).

Am Beispiel der Kategorie Geschlecht, die - wie auch die Kategorie Klasse/Schicht - alle gesellschaftlichen Individuen umfasst, und der Kategorie Behinderung, die sich im Vergleich dazu - wie auch die im schulischen Bereich gängige Kategorie des Migrationshintergrunds - nur auf eine gesellschaftliche Minderheit bezieht, soll dies exemplarisch dargelegt werden.

Bezogen auf die Binnenstruktur der Kategorie Geschlecht sind im Bereich des Bildungswesens unterschiedliche, tendenziell konkurrierende Positionen zu finden: Die starre (protonormalistische) Konstruktion der Zweigeschlechtlichkeit (entweder männlich oder weiblich) mit entsprechenden Charakter- und Verhaltenszuweisungen an Mädchen und Jungen wird in der pädagogischen Theorie und Praxis mancherorts (noch) vertreten, obwohl immer deutlicher wird, dass durch sie Mädchen und Jungen in ihren Entwicklungs- und Entfaltungsmöglichkeiten eingeschränkt werden. Dagegen werden vermehrt flexiblere (flexibelnormalistische) Konstruktionen von Geschlecht bewusst wahrgenommen, erprobt und kritisch überprüft; etwa in dem von Annedore Prengel (2011) für den Elementarbereich des Bildungswesens beschriebenen Sinne: „Der Überblick über die widersprüchlichen Forschungsbefunde zeigt, dass Kinder - holzschnittartig verkürzend formuliert vier unterschiedliche Konstruktionsweisen von Geschlecht entwickeln: Sie stellen 'dualistisch' die Zweigeschlechtlichkeit, 'universalistisch' die Gemeinsamkeiten zwischen beiden Geschlechtern oder 'pluralistisch' die Vielfalt innerhalb jedes Geschlechts und 'transversal' die Überschneidungen zwischen beiden Geschlechtern in den Vordergrund (Prengel 2009)“" (Prengel 2011: 2). Schließlich entsteht ein Bewusstsein darüber, dass (im transnormalistischen Sinne) auch Geschlechtsvarianz und Transidentität (vgl. Brill \& Pepper 2011) als individuelle und gesellschaftliche Realitäten zur Kenntnis zu nehmen und im Sinne einer inklusiven Pädagogik für alle Kinder anzuerkennen und positiv zu nutzen sind.

Für die Binnenstruktur der Kategorie Behinderung und deren Funktionen im Bereich des Bildungswesens ist vergleichend festzustellen: Behinderung ist eine Kategorie, unter der eine gesellschaftliche Minderheit (in Deutsch- 
land von durchschnittlich ca. 10 Prozent) zusammengefasst wird, deren Leistungsfähigkeit (fiktiv oder real, differenziert nach Alter und Geschlecht) im Vergleich zur durchschnittlichen Leistungsfähigkeit der Gesamtbevölkerung auf Grund gesundheitlicher Einschränkungen als beeinträchtigt gilt. Im protonormalistischen Sinne findet in der Gesamtgesellschaft eine Differenzierung zwischen der Mehrheitsgesellschaft/Normalbevölkerung und der gesellschaftlichen Minderheit der behinderten Menschen statt bzw. im traditionellen Bildungswesen (vor allem im Bereich der Schule) die Differenzierung zwischen Regel- und Sonderschüler*innen. Eine Flexibilisierung dieser starren Gegenüberstellung von normal und behindert fand im Bildungswesen vor allem in den 1990er Jahren (beeinflusst durch die Forderungen der Integrationspädagogik) statt, die u.a. in der Überwindung der Konstruktion des Sonderschulaufnahmeverfahrens (SAV) durch die Konstruktion des ,Sonderpädagogischen Förderbedarfs“ einschließlich der Flexibilisierung der möglichen Förderorte (Förder-/Sonderschule oder Regelschule) sichtbar wurde. $\mathrm{Zu}$ dieser Flexibilisierung gehörten u.a. auch die Einrichtung von Sonderklassen an Regelschulen und ähnliche additive Annäherungsformen zwischen Regelund Sonderpädagogik. Im Sinne einer inklusiven Pädagogik ist jedoch erst wie schon in den wissenschaftlich fundierten Ansätzen der Integrationspädagogik transnormalistisch orientiert - die gemeinsame Förderung und Unterrichtung aller Kinder anzusehen, die auch die Frage auf den Plan ruft, ob dann überhaupt noch einzelnen von ihnen ein individueller ,sonderpädagogischer Förderbedarf" attestiert werden müsse (Stichwort: „Etikettierung als Gefahr oder Chance?““; vgl. exemplarisch Preuss-Lausitz 2011: 165ff.).

Auch für die anderen bildungsrelevanten Strukturkategorien - Alter, Klasse/Schicht/soziale Lage, Ethnizität/Migrationshintergrund - ist von entsprechend differenzierten Binnenstrukturen auszugehen (vgl. Autorengruppe Bildungsberichterstattung 2012; 2014; Wansing \& Westphal 2014), die jedoch an dieser Stelle nicht näher ausgeführt werden können.

Die beiden näher ausgeführten Beispiele, Geschlecht und Behinderung, zeigen exemplarisch, dass jede soziale Strukturkategorie über ihre eigenen Binnenstrukturen verfügt, die Beachtung finden sollten, wenn es - im darauf folgenden analytischen Schritt - darum geht, gegenseitige Beeinflussungen und Wechselwirkungen zwischen einzelnen Strukturkategorien zu erkennen.

\section{Zielperspektive: Reflexive Inklusion - Schwerpunkt: Professionelle Anforderungen an Pädagog*innen}

Vor dem dargestellten Hintergrund wird deutlich, dass eine zu entwickelnde (reflexive) inklusive Pädagogik vor großen Herausforderungen steht. Diese können nach Ansicht der Verfasserin des vorliegenden Beitrages nur bewäl- 
tigt werden, wenn das Grundanliegen der inklusiven Pädagogik mit einer entsprechenden positiven Wertschätzung angenommen wird. Dazu bedarf es allerdings einer „umfassenden Inklusionsstrategie“ (Stein 2015: 405; in Anlehnung an Hanesch 2006) in der Gesamtgesellschaft, die weit über die Pädagogik hinaus reicht, aber dennoch zentral auf die Pädagogik angewiesen ist. Damit verbunden ist die Debatte um eine neue, inklusionspädagogische Professionalität der beteiligten Pädagog*innen,

„[...] die mit den Konstruktionen von Regel- und Sonderpädagogik nicht vereinbar ist [...] denn die neue Ausrichtung an einem positiv konnotierten Umgang mit Heterogenität steht der traditionellen Orientierung an der Unterrichtung möglichst homogener Gruppen von Kindern und Jugendlichen in einem entsprechend hierarchisch organisierten Bildungswesen diametral entgegen" (Schildmann 2015b: 21).

Die Genderperspektive in der Inklusiven Pädagogik ist eine Perspektive unter mehreren und kommt vor allem in Wechselwirkung mit sozialer Lage (Klasse), kulturellem Hintergrund sowie mit den Konstruktionen von Normalität und Behinderung zum Ausdruck. Die zu reflektierenden Fragen sind auf den unterschiedlichen Ebenen des gesellschaftlichen Miteinanders angesiedelt. Bewährt hat sich bereits im Rahmen der integrationspädagogischen Forschung (vgl. Schildmann 1996 \& 2012 in Anlehnung an die Forschungsgruppe um Helmut Reiser; vgl. Klein et al. 1987) folgendes Ebenenmodell: innerpsychische, interaktionelle, institutionelle Ebene und gesamtgesellschaftliche Ebene.

Auf der innerpsychischen Ebene stehen die eigenen Konzeptionen von „Gender/Geschlecht“ der Pädagog*innen im Elementar-, Primar- und Sekundarbereich des Bildungswesens zur Debatte (vgl. dazu Schildmann 2012: 31): Wie reflektieren Pädagog*innen ihre eigene geschlechterspezifische Sozialisation und Identitätsarbeit (für die Integrationspoädagogik vgl. Nagode 2002)? Und wie begegnen sie der Identitätsarbeit der ihnen anvertrauten Mädchen und Jungen mit deren je eigenem „Bildungsprojekt Geschlecht“ (Rabe-Kleberg 2006) unter verschiedensten sozialen, kulturellen und individuellen Rahmenbedingungen?

Auf der interaktionellen Ebene (vgl. nochmals Nagode 2002) geht es, bezogen auf die Kategorie Geschlecht, vor allem um das „,doing gender“ in der Inklusiven Pädagogik. Hier ist z.B. auf die strukturellen Wechselwirkungen einzugehen, die sich zeigen zwischen den unterschiedlichen sozialen Verhältnissen, kulturellen Bindungen und Vorstellungen, geschlechtlichen Identitätskonstruktionen (auch jenseits der gängigen sozialen Zweigeschlechtlichkeit) sowie individuellen Fähigkeiten und ggf. Beeinträchtigungen (vgl. Schildmann 2012: 32). Auf dieser Ebene experimentieren Kinder und Jugendliche mit ihren Geschlechterrollen, wenn die Möglichkeit im Rahmen der inklusiven Erziehung dazu gegeben wird. Das schließt jedoch auch ein, dass gesellschaftliche Machtstrukturen zwischen den Geschlechtern reflek- 
tiert werden, die die Jungen und Mädchen an anderen Orten kennenlernen. Hier sollte aber auch die Frage behandelt werden, wie die konkreten Lebenswelten und Interessen der beteiligten Mädchen und Jungen ihren Niederschlag im Unterricht finden können.

Mit den interaktionellen Perspektiven verknüpft ist die institutionelle Ebene der inklusiven Pädagogik und damit auch die Curriculum- und Lehrplanentwicklung (vgl. exemplarisch Ziemen 2003; Platte 2004):

„Zu fragen ist (wie schon im Rahmen der Integrationspädagogik), ob die allgemein geltenden Lehrpläne der Regel- und Sonderpädagogik des hierarchischen Bildungswesens die Anforderungen erfüllen, die eine Inklusive Pädagogik stellt, 'oder ob vielmehr neue Ziele und Inhalte formuliert werden müßten, um die Differenzierung im Sinne des Heterogenitätsprinzips einlösen zu können` (Schildmann 1996: 85)““(Schildmann 2012: 33).

Eng verbunden mit allen drei genannten Ebenen, auf denen in den letzten Jahren eine enorme Dynamik zu verzeichnen ist, sind Fragen, die die Konstruktionen der Gesamtgesellschaft betreffen und die von Pädagog*innen reflektiert werden müssen, aber von ihnen allein nicht gelöst werden können. Dazu gehört neben anderen vor allem die kritische Auseinandersetzung mit den gesellschaftlichen Leistungsprinzipien: Sind diese (im protonormalistischen Sinne) unhinterfragt zu akzeptieren oder können sie (im flexibel normalistischen Sinne) aufgeweicht werden oder sogar (im transnormalistischen Sinne) - zumindest für den Bereich der Bildung, wie von Integrations- und Inklusionsforscher*innen gefordert und versucht - durchbrochen werden? Auch diese Auseinandersetzung ist Teil der professionellen (Selbst)Reflexion von Pädagog*innen; denn sie wirkt auf die anderen Reflexionsebenen ein bzw. zurück.

Wie der vorliegende Beitrag zu zeigen versucht hat, sind auf dem Weg von der (reflexiven) Koedukation zur (reflexiven) Inklusion Problemstellungen zu meistern, die sich - im Sinne des sportlichen Wettkampfes - auch als ein komplizierter Hürdenlauf vorstellen lassen:

Zum einen sind die Hürden, wie am Anfang dieses Kapitels gezeigt wurde, auf unterschiedlichen analytischen Ebenen (der innerpsychischen, interaktionellen, institutionellen, gesamtgesellschaftlichen) angesiedelt. Zum zweiten ist bei der Bewältigung der anstehenden Aufgaben zu bedenken, dass nicht nur eine Teildisziplin der Pädagogik diesen Entwicklungsprozess hin zu einer reflexiven inklusiven Pädagogik stemmen kann, sondern alle (oder zumindest mehrere) daran beteiligt sein müssen, wobei zu bedenken ist, dass sie mit jeweils unterschiedlichen historischen und systematischen Voraussetzungen, Motivationen und Perspektiven aufeinandertreffen, um die große Bildungsreform in Angriff zu nehmen. Für die pädagogischen Reformströmungen der feministischen Pädagogik, der interkulturellen Pädagogik und der integrativen Pädagogik, deren professionelles Wissen im Zuge der Umsetzung einer reflexiven inklusiven Pädagogik an vorderster Stelle gefragt 
sein dürfte, hat Annedore Prengel bereits vor 25 Jahren in ihrer „Pädagogik der Vielfalt" (1993a) gezeigt, welche (vergleichbaren) historischen Stadien diese durchlaufen haben und welche strukturellen Gemeinsamkeiten sie aufweisen, auch wenn bis zum Ende des 20. Jahrhunderts nur wenige engere Verbindungen und direkte Kooperationen zwischen ihnen eingegangen wurden. Die konstruktive Zusammenarbeit steht ihnen - wie auch den traditionellen Teildisziplinen der Erziehungswissenschaft - also weitgehend noch bevor. Um sie erfolgreich zu gestalten, wird schließlich ein strukturierter Reflexionsprozess notwendig sein, der sich mit den unterschiedlichen Konstruktionen von Normalität und Abweichung in den Teildisziplinen ebenso wie mit den Wechselwirkungen/Intersektionen (vgl. exemplarisch Walgenbach $2016^{8}$ ) zwischen den für die inklusive Pädagogik relevanten sozialen Ungleichheitslagen der Kinder und Jugendlichen beschäftigt.

Die mit den genannten Problemstellungen verbundenen Hürden müssen erkannt, benannt und reflektiert werden, bevor sie erfolgreich überwunden werden können.

\section{Literatur}

Autorengruppe Bildungsberichterstattung (2012): Bildung in Deutschland 2012. Ein indikatorengestützter Bericht mit einer Analyse zur kulturellen Bildung im Lebenslauf. Bielefeld: Bertelsmann Verlag.

Autorengruppe Bildungsberichterstattung (2014): Bildung in Deutschland 2014. Ein indikatorengestützter Bericht mit einer Analyse zur Bildung von Menschen mit Behinderungen. Bielefeld: Bertelsmann Verlag.

Brill, Stephanie/Pepper, Rachel (2011): Wenn Kinder anders fühlen - Identität im anderen Geschlecht. München: Ernst Reinhardt Verlag.

Budde, Jürgen/Hummrich, Merle (2013): Reflexive Inklusion. In: Zeitschrift für Inklusion online 4, S. 1-6. http://www.inklusiononline.net/index.php/inklusion-online/article/view/193/199 [Zugriff: 23.11.2015].

Degener, Theresia/Diehl, Elke (Hrsg.) (2015): Handbuch Behindertenrechtskonvention. Teilhabe als Menschenrecht - Inklusion als gesellschaftliche Aufgabe. Bonn: Bundeszentrale für politische Aufklärung.

Faulstich-Wieland, Hannelore (1994): Reflexive Koedukation. Zur Entwicklung der Koedukationsdebatte in den Bundesländern. In: Bracht, Ul-

8 Die von Walgenbach (2016: 654) kurz umrissene Position zum Verhältnis zwischen inklusiver Pädagogik und Intersektionalitätsforschung ist diskussionswürdig; sie wird von der Verfasserin des vorliegenden Beitrags nicht uneingeschränkt geteilt (vgl. Schildmann 2012). 
la/Keiner, Dieter (Hrsg.): Jahrbuch für Pädagogik 1994: Geschlechterverhältnisse und die Pädagogik. Frankfurt a.M.: Peter Lang, S. 325-342.

Faulstich-Wieland, Hannelore (2011): Einleitung. In: Dies. (Hrsg.): Umgang mit Heterogenität und Differenz. Professionswissen für Lehrerinnen und Lehrer. Baltmannsweiler: Schneider Verlag Hohengehren, S. 9-20.

Faulstich-Wieland, Hannelore/Horstkemper, Marianne (2012): Schule und Genderforschung. In: Kampshoff, Marita/Wiepcke, Claudia (Hrsg.): Handbuch Geschlechterforschung und Fachdidaktik. Wiesbaden: Springer VS, S. 25-38.

Feuser, Georg (1986): Unverzichtbare Grundlagen und Formen der gemeinsamen Erziehung behinderter und nichtbehinderter Kinder in Kindergarten und Schule. In: Behindertenpädagogik 25, 2, S. 122-139.

Feuser, Georg (1989): Allgemeine integrative Pädagogik und entwicklungslogische Didaktik. In: Behindertenpädagogik 28, 1, S. 4-48.

Hinz, Andreas (1993): Heterogenität in der Schule. Integration - Interkulturelle Erziehung - Koedukation. Hamburg: Tectum.

Kampshoff, Marita/Wiepcke, Claudia (Hrsg.) (2012): Handbuch Geschlechterforschung und Fachdidaktik. Wiesbaden: Springer VS.

Kelle, Helga/Tervooren, Anja (Hrsg.) (2008): Ganz normale Kinder. Heterogenität und Standardisierung kindlicher Entwicklung. Weinheim/München: Juventa.

Kiuppis, Florian (2014): Heterogene Inklusivität, inklusive Heterogenität. Bedeutungswandel imaginierter pädagogischer Konzepte im Kontext Internationaler Organisationen. Münster: Waxmann.

Klein, Gabriele u.a. (1987): Integrative Prozesse in Kindergartengruppen. Über die gemeinsame Erziehung von behinderten und nichtbehinderten Kindern. Weinheim und München: DJI.

Kreienbaum, Maria Anna (2004): Schule: Zur reflexiven Koedukation. In: Becker, Ruth/Kortendiek, Beate (Hrsg.): Handbuch Frauen- und Geschlechterforschung. Wiesbaden: VS Verlag für Sozialwissenschaften, S. $582-589$.

Link, Jürgen (1997): Versuch über den Normalismus. Wie Normalität produziert wird. Opladen: Westdeutscher Verlag.

Marks, Dana-Kristin (2011): Konstruktionen von Behinderung. Unter besonderer Berücksichtigung der Strukturkategorie Geschlecht. Bochum/Freiburg: Projekt Verlag.

Merz-Atalik, Kerstin (2001): Interkulturelle Pädagogik in Integrationsklassen. Opladen: Leske + Budrich.

Moser, Vera/Schäfer, Lea/Redlich, Hubertus (2011): Kompetenzen und Beliefs von Förderschullehrkräften in inklusiven Settings. In: Lütje-Klose, Birgit u.a. (Hrsg.): Inklusion in Bildungsinstitutionen. Bad Heilbrunn/Obb.: Klinkhardt, S. 143-149. 
Moser, Vera/Roll, Mathias/Seidel, Carola (2006): Geschlechterinszenierungen in der Sonderschule. In: Vierteljahresschrift für Heilpädagogik und ihre Nachbargebiete (VHN) 75, 4, S. 305-316.

Nagode, Claudia (2002): Grenzenlose Konstruktionen - konstruierte Grenzen? Behinderung und Geschlecht aus Sicht von Lehrerinnen in der Integrationspädagogik. Münster: LIT.

Platte, Andrea (2004): Schulische Lebens- und Lernwelten gestalten. Didaktische Fundierung inklusiver Bildungsprozesse. Münster: Monsenstein und Vannerdat.

Platte, Andrea (2015): Inklusive Bildung: Leitidee von der Kindertageseinrichtung bis zur Hochschule. In: Degener, Theresia/Diehl, Elke (Hrsg.): Handbuch Behindertenrechtskonvention. Teilhabe als Menschenrecht Inklusion als gesellschaftliche Aufgabe. Bonn: Bundeszentrale für politische Aufklärung.

Popp, Ulrike (2011): Bildung der Geschlechter - geschlechterdifferente Bildung? In: Faulstich-Wieland, Hannelore (Hrsg.): Umgang mit Heterogenität und Differenz. Professionswissen für Lehrerinnen und Lehrer. Baltmannsweiler: Schneider Verlag Hohengehren, S. 73-96.

Prasad, Nivedita (2015): Entweder Schwarz oder weiblich? Zum Umgang mit Intersektionalität in UN-Fachausschüssen. In: Attia, Iman u.a. (Hrsg.): Dominanzkultur reloaded. Neue Texte zu gesellschaftlichen Machtverhältnissen und ihren Wechselwirkungen. Bielefeld: Transcript, S. 129-142.

Prengel, Annedore (1993a): Pädagogik der Vielfalt. Verschiedenheit und Gleichberechtigung in Interkultureller, Feministischer und Integrativer Pädagogik. Opladen: Leske + Budrich.

Prengel (1993b): Sind Mädchen die Integrationshelferinnen par excellence? Mädchen im Modernisierungsprozeß. In: Gehrmann, Petra/Hüwe, Birgit (Hrsg.): Forschungsprofile der Integration von Behinderten. Essen: Neue deutsche Schule, S. 54-62.

Prengel, Annedore (1999): Vielfalt durch gute Ordnung im Anfangsunterricht. Opladen: Leske + Budrich.

Prengel, Annedore (2009): Vielfalt. In: Dederich, Markus/Jantzen, Wolfgang (Hrsg.): Behinderung, Bildung, Partizipation. Enzyklopädisches Handbuch der Behindertenpädagogik, Bd. 2. Stuttgart: Kohlhammer, S. 105112.

Prengel, Annedore (2011): Ziele einer „geschlechterdemokratischen“ Pädagogik im inklusiven Kindergarten. In: Zeitschrift für Inklusion, Inklusion-Online 1, S. 7. http://www.inklusion-online.net/index.php/inklusiononline/article/view/108/108 [Zugriff: 23.11.1015].

Prengel, Annedore (2015): Pädagogik der Vielfalt: Inklusive Strömungen in der Sphäre spätmoderner Bildung. In: Erwägen Wissen Ethik 26, 2, S. 157-168 \& S. 274-286 (Replik). 
Preuss-Lausitz, Ulf (1998): Auch Behinderte sind Jungen und Mädchen. Neue geschlechtsspezifische Erkenntnisse zur gemeinsamen Erziehung. In: Zeitschrift für Heilpädagogik 49, 5, S. 216-225.

Preuss-Lausitz, Ulf (2011): Integration und Inklusion von Kindern mit Behinderungen - Ein Weg zur produktiven Vielfalt in einer gerechten Schule. In: Faulstich-Wieland, Hannelore (Hrsg.): Umgang mit Heterogenität und Differenz. Professionswissen für Lehrerinnen und Lehrer. Baltmannsweiler: Schneider Verlag Hohengehren, S. 161-180.

Rabe-Kleberg, Ursula (2006): Gender als Bildungsprojekt. Wie Mädchen und Jungen sich die zweigeschlechtliche Welt aneignen. In: Betrifft Mädchen 19, 3, S. 100-104.

Redlich, Hubertus u.a. (Hrsg.) (2015): Veränderung und Beständigkeit in Zeiten der Inklusion. Perspektiven Sonderpädagogischer Professionalisierung. Bad Heilbrunn/Obb.: Klinkhardt.

Rohr, Barbara (1980): Koedukation und Koinstruktion in der Schule für Lernbehinderte. In: Baier, Herwig/Klein, Gerhard (Hrsg.): Die Schule für Lernbehinderte. Organisatorische Fragen pädagogisch gesehen. Berlin: Carl Marhold, S. 217-235.

Schildmann, Ulrike (1996): Integrationspädagogik und Geschlecht. Theoretische Grundlegung und Ergebnisse der Forschung. Opladen: Leske + Budrich.

Schildmann, Ulrike (1997): Integrationspädagogik und Normalisierungsprinzip - ein kritischer Vergleich. In: Zeitschrift für Heilpädagogik 48, 3, S. 90-96.

Schildmann, Ulrike (1999): Koedukation. In: Konrad Bundschuh u.a. (Hrsg.): Wörterbuch Heilpädagogik. Bad Heilbrunn/Obb.: Klinkhardt, S. 165167. 2., durchgesehene Auflage 2002; S. 163-165.

Schildmann, Ulrike (2000): Koedukation in der Integrationspädagogik. In: Gemeinsam leben 8, 4, S. 167-172.

Schildmann, Ulrike (2004): Normalismusforschung über Behinderung und Geschlecht. Eine empirische Untersuchung der Werke von Barbara Rohr und Annedore Prengel. Opladen: Leske + Budrich.

Schildmann, Ulrike (2009): Normalität. In: Dederich, Markus/Jantzen, Wolfgang (Hrsg.): Behinderung, Bildung, Partizipation. Enzyklopädisches Handbuch der Behindertenpädagogik, Bd. 2. Stuttgart: Kohlhammer, S. 204-208.

Schildmann, Ulrike (2011): Koedukativer Unterricht. In: Astrid Kaiser u.a. (Hrsg.): Didaktik und Unterricht. Enzyklopädisches Handbuch der Behindertenpädagogik, Bd. 4. Stuttgart: Kohlhammer, S. 232-236.

Schildmann, Ulrike (2011a): Verhältnisse zwischen Geschlecht, Behinderung und Alter/Lebensabschnitten als intersektionelle Forschungsperspektive. In: Journal Netzwerk Frauen- und Geschlechterforschung NRW Nr. 29, S. 13-15. 
Schildmann, Ulrike (2012): Die Genderperspektive in der Inklusiven Pädagogik. In: Zeitschrift für Inklusion online, 3, Druckversion: S. 30-38. http://www.inklusion-online.net/index.php/inklusiononline/article/view/42/42 [Zugriff: 23.11.2015].

Schildmann, Ulrike (2012a): Verhältnisse zwischen Inklusiver Pädagogik und Intersektionalitätsforschung: sieben Thesen. In: Seitz, Simone/Finner, Nina-Kathrin (Hrsg.): Inklusiv gleich gerecht? Inklusion und Bildungsgerechtigkeit. Bad Heilbrunn/Obb.: Klinkhardt, S. 93-99.

Schildmann, Ulrike (2013): Rezension zu Marita Kampshoff und Claudia Wiepcke (Hrsg.) (2012): Handbuch Geschlechterforschung und Fachdidaktik. In: Journal Netzwerk Frauen- und Geschlechterforschung NRW Nr. 32, S. 89-91.

Schildmann, Ulrike (2013a): Wechselwirkungen zwischen Geschlecht und Behinderung von der frühen Kindheit bis ins hohe Erwachsenenalter. In: Behindertenpädagogik 52, 1, S. 68-81.

Schildmann, Ulrike (2015): Normativität und Normalität außer Balance. Stellungnahme zu Annedore Prengel: „Pädagogik der Vielfalt. Inklusive Strömungen in der Sphäre spätmoderner Bildung“. In: Erwägen Wissen Ethik (EWE) 26, 2, S. 254-256.

Schildmann, Ulrike (2015a): Institutionelle Übergänge in der gesamten Lebensspanne unter besonderer Berücksichtigung von Behinderung und Geschlecht. In: VHN 84, 2, S. 93-97.

Schildmann, Ulrike (2015b): Sonderpädagogische und inklusionspädagogische Professionalität - ein kritischer Vergleich. In: Redlich, Hubertus/Schäfer, Lea/Wachtel, Grit/Zehbe, Katja/Moser, Vera (Hrsg.): Veränderung und Beständigkeit in Zeiten der Inklusion. Perspektiven Sonderpädagogischer Professionalität. Bad Heilbrunn/Obb.: Klinkhardt, S. 18-24.

Stechow, Elisabeth von (2004): Erziehung zur Normalität. Eine Geschichte der Ordnung und Normalisierung der Kindheit. Wiesbaden: VS Verlag für Sozialwissenschaften.

Stechow, Elisabeth von (2008): Zur Geschichte der Idee eines „normalen Verhaltens“. In: Kelle, Helga/Tervooren, Anja (Hrsg.): Ganz normale Kinder. Heterogenität und Standardisierung kindlicher Entwicklung. Weinheim/München: Juventa, S. 75-91.

Walgenbach, Katharina (2016): Intersektionalitätsforschung. In: Hedderich, Ingeborg u.a. (Hrsg.): Handbuch Inklusion und Sonderpädagogik. Bad Heilbrunn/Obb.: Klinkhardt, S. 650-655.

Wansing, Gudrun/Westphal, Manuela (Hrsg.) (2014): Behinderung und Migration. Inklusion, Diversität, Intersektrionalität. Wiesbaden: Springer VS.

Wenning, Norbert (1999): Vereinheitlichung und Differenz. Zu den ,wirklichen" gesellschaftlichen Funktionen des Bildungswesens im Umgang mit Gleichheit und Verschiedenheit. Opladen: Leske + Budrich. 
Ziemen, Kerstin (2003): Integrative Pädagogik und Didaktik. Aachen: Shake. 